\title{
Linx
}

Revue des linguistes de l'université Paris X Nanterre

66-67| 2012

Dynamiques de la construction des sens attendus et inattendus dans les langues

\section{Even et quelques traductions en français : la référence à l'inattendu}

\section{Agnès Leroux}

\section{(2) OpenEdition}

\section{Journals}

Édition électronique

URL : https://journals.openedition.org/linx/1444

DOI : $10.4000 / \operatorname{linx} .1444$

ISSN : 2118-9692

Éditeur

Presses universitaires de Paris Nanterre

Édition imprimée

Date de publication : 15 septembre 2012

Pagination : $35-50$

ISSN : 0246-8743

Référence électronique

Agnès Leroux, «Even et quelques traductions en français : la référence à l'inattendu », Linx [En ligne], 66-67 | 2012, mis en ligne le 15 septembre 2013, consulté le 21 septembre 2021. URL : http:// journals.openedition.org/linx/1444 ; DOI : https://doi.org/10.4000/linx.1444 


\title{
Even et quelques traductions en français : la référence à l'inattendu
}

\author{
Agnès Leroux UA 370 (CREA GReG) \\ Université Paris Ouest Nanterre la Défense
}

\section{Introduction}

Dans cet article, nous allons nous intéresser à la façon dont even permet de référer à l'inattendu ou de construire un sens inattendu, à l'aide du cadre théorique de la Théorie des Opérations Énonciatives d'Antoine Culioli (désormais TOE).

Si nous proposons une étude dans le cadre de la TOE, cela implique que l'analyse ne portera que sur énoncés authentiques. Mais au sein de cette étude contrastive anglais/français, certains exemples seront traduits de l'anglais vers le français. Les traductions sont issues d'ouvrages publiés. Il ne s'agira cependant pas d'une présentation prescriptive, qui prétendrait jauger les traductions proposées pour l'adverbe even et déterminer celles qui sont souhaitables. Bien au contraire, la définition d'un schème opérationnel pour even sera l'occasion de mettre en évidence les paramètres de construction du sens qui permettent (et non qui obligent) de traduire en français par un marqueur plutôt que par un autre. Tout l'intérêt de la partie contrastive résidera d'ailleurs dans la comparaison du fonctionnement des marqueurs français en fonction de la construction du sens dans les énoncés en anglais, et plus particulièrement des différentes façons dont le traducteur a conservé la référence à l'inattendu.

Dans un premier temps l'analyse sera unilingue, sur l'anglais, afin de poser clairement le schème opérationnel dont even est la trace. Nous passerons ensuite à 
Agnès Leroux

l'observation des traductions possibles que nous étudierons à travers le filtre de l'opération marquée selon nous par even.

Le mot even a deux natures différentes, puisqu'il est soit adjectif, soit adverbe. Nous nous intéresserons exclusivement à l'adverbe even. Il est cependant impossible de ne pas s'arrêter sur le sens de l'adjectif, élément du domaine lexical, afin de mieux cerner le sens construit par le marqueur de nature adverbiale : We're even. The ground is even.

Le sens de even adjectif est construit de la façon suivante: soit le sujet grammatical renvoie à un élément qui est homogène, dont toutes les parties sont identiques, soit il renvoie à au moins deux éléments qui sont considérés comme étant au même niveau.

Il nous semble que, si simples et courts et hors contexte soient-ils, ces énoncés permettent de construire un sens induit : s'il est nécessaire de dire qu'à un instant T les choses sont égales, c'est parce que, soit à un instant préalable, soit selon une préconstruction de l'énonciateur, elles ne le sont pas. Un état de fait vient remettre en question une inégalité originelle. En d'autres termes, une occurrence permet d'égaliser ce qui, à l'origine, était disparate ou pas aussi homogène qu'attendu ou souhaitable. Nous posons par conséquent l'hypothèse selon laquelle l'adverbe even marque un hiatus entre deux situations : une préalable ou un préconstruit, et une effective.

Nous construirons par conséquent l'étude de l'inattendu dans cet article comme l'observation d'un marqueur de hiatus en anglais, et de quelques-unes de ses traductions possibles en français, entre l'attendu et l'effectif, entre le préconstruit, 'préalable', et ce qui est effectivement le cas.

\section{Even adverbe : ce que devient la mise à niveau}

Lorsque even est adverbe, il peut se placer devant un nom, un verbe ou un adjectif, c'est ce qu'on appelle un 'focus adverb' (Close 1975, p. 287-288) ou adverbe focalisant ${ }^{1}$, c'est-à-dire un adverbe qui permet de mettre en relief un seul élément à l'intérieur d'une proposition. R. A. Close précise qu'even est un exemple parfait de 'focus adverb', qui signifie que l'énonciateur ajoute un élément à une liste constituée au préalable. Pour définir le schème opérationnel dont il est la marque, nous limiterons notre analyse unilingue au placement devant un nom, un verbe ou un adjectif comparatif, emplois qui correspondent à des traductions diversifiées et intéressantes en français.

Pour la cohérence de cette présentation, une parenthèse sur notre conception de la formation d'un énoncé s'impose ici. Dans un souci de simplicité nous ne décrirons que des énoncés simples, selon le schéma canonique Sujet-Verbe-Complément :

L'énoncé se forme en trois niveaux simultanés mais bien distincts :

- un niveau notionnel, niveau auquel se définissent les éléments utilisés par l'énonciateur : au moins deux notions, au plus trois dans cette description. Une notion est un faisceau de propriétés qui permet de concevoir ou de comprendre de quel élément du domaine de référence il est question. L'ensemble de ces notions construit une lexis ; nous pouvons prendre pour exemple la lexis /souris, chat, manger/.

${ }^{1}$ Ma traduction. 
- Au niveau prédicatif, la lexis est orientée : on choisit le terme de départ, et le terme d'arrivée-autrement dit, qui fait quoi : le plus simple avec la lexis que nous avons décidé d'utiliser à titre d'exemple est $<$ chat - manger - souris $>$. Ceci étant si nous nous plaçons dans le domaine de référence du dessin animé, nous pouvons très bien l'orienter ainsi $<$ souris - manger - chat $>$. De façon plus générale nous avons alors $\left.<\mathrm{C}_{0}-\mathrm{R}-\mathrm{C}_{1}\right\rangle$, un complément de rang 0 mis en relation avec un complément de rang 1. cette notation permet de différencier le sujet réel, l'agent, du sujet grammatical, qui peut être différent dans un énoncé à la voix passive.

- Au niveau énonciatif, la relation prédicative est déterminée et est assortie de tous les marqueurs nécessaires à son repérage par rapport à une situation d'énonciation: Hier le chat a mangé une souris, Les chats ça mange des souris, Ta souris a été mangée (par le chat), etc. Tous ces exemples déterminés et assortis de marqueurs (temps, aspect, mode, articles, adverbe, etc.) représentent autant d'occurrences de la relation prédicative, désormais RP dans cet article. Chaque occurrence d'une RP s'insère ou pas, selon l'énonciateur, dans un domaine notionnel, c'est-à-dire dans une représentation conceptuelle de ce à quoi doit correspondre une occurrence donnée.

Par situation d'énonciation nous entendons la mise en place des coordonnées nécessaires à l'émission d'un énoncé, à savoir un énonciateur (tout sujet qui parle) et un moment d'énonciation. Ces deux coordonnées sont reprises sous la forme Sit $(\mathrm{S}, \mathrm{T})$, la situation d'énonciation origine étant $\mathrm{Sit}_{0}\left(\mathrm{~S}_{0}, \mathrm{~T}_{0}\right)$.

Dans cette partie unilingue anglais, nous allons nous attacher à montrer que le schème opérationnel marqué par even est le suivant: even est la trace d'une mise à niveau entre un préalable selon l'énonciateur, désormais $\mathrm{S}$ dans cet article, et l'effectif constaté dans la situation d'énonciation ou d'énoncé, globalement désigné par la lettre $\mathrm{T}$ ici. Cette opération permet d'exprimer le caractère inattendu d'un état de fait, de ce qui est le cas. Cette opération est cohérente avec le sens de even tel qu'avancé par R. A. Close, puisque la liste préalable se trouve modifiée, mise à niveau, par un état de fait.

\subsection{Even adverbe devant un nom}

Dans les énoncés suivants, le sens de even, tel que défini précédemment, transparait clairement : even, placé devant un nom en position de $\mathrm{C}_{0}$ ou de $\mathrm{C}_{1}$, permet une mise à niveau ponctuelle, donc pour une seule occurrence de la relation prédicative, entre la liste des possibles et celle de ce qui est le cas :

Exemple 1: And in a surprising twist that shocked even his own lawyer, Shenkman also made a statement: 'I view myself right now as a prisoner of war, a POW. In July 2006, Nancy Tyler declared war. She fired the first shot in the famous war of the Tylers by filing for divorce.' In over 40 years of practice, I have never seen a criminal defendant say anything like what I heard Richard say to Judge Dewey and the court. (COCA, corpus électronique)

[Et lors d'un retournement surprenant qui a choqué son propre avocat lui-même, Shenkman a également fait une déclaration : 'Je me considère désormais comme un prisonnier de guerre. En juillet 2006, Nancy Tyler a déclaré la guerre. Elle a tiré le premier coup de la 
Agnès Leroux

célèbre guerre des Tylers lorsqu'elle a déposé sa demande de divorce.' En plus de 40 ans de pratique, je n'ai jamais vu un accusé faire une déclaration similaire à celle de Richard au Juge Dewey et à la Cour. (Notre traduction)]

Exemple 2: Nancy's sister Marilyn says even her closest family members had no idea what Shenkman was doing to her. He kept her isolated. (COCA, corpus électronique)

[La sœur de Nancy, Marilyn, dit que même la famille proche n'avait aucune idée du comportement de Shenkman envers elle. Il la maintenait isolée. (Notre traduction)]

Exemple 3: You take the test and you find that even people of color are more likely to ascribe negative attributes to darker skinned people. (COCA, corpus électronique)

Regardez les résultats du test et vous vous rendrez compte que même les gens de couleur vont plus facilement associer des caractéristiques négatives à des gens dont la peau est plus foncée. (Notre traduction)]

Dans ces énoncés, nous pouvons isoler les relations prédicatives (désormais $\mathrm{RP})$ suivantes sur le modèle $<\mathrm{C}_{0}-\mathrm{R}-\mathrm{C}_{1}>$ :

1) < twist - shock lawyer > even porte sur lanyer et ses déterminations ;

2) < family member - have no idea > even porte sur family member et ses déterminations;

3) < people of color - be more likely...> even porte people of color.

Parmi les éléments possibles pour instancier les places de $\mathrm{C}_{0}$ ou $\mathrm{C}_{1}, \mathrm{X}$, l'élément effectif, est considéré comme n'ayant pas a priori toutes les propriétés pour valider la relation prédicative. Il est à l'extérieur des possibles selon $\mathrm{S}$; dans l'explicitation suivante, nous utiliserons l'adverbe normalement pour signifier la prise en compte d'un préalable pour S :

- en 1, normalement, l'avocat de la personne jugée est au courant de la stratégie de l'accusé ;

- en 2, normalement les membres les plus proches de la famille sont au courant s'il se passe quelque chose de grave ;

- en 3 normalement les gens de couleur ont moins de préjugés raciaux que les blancs.

En revanche, pour ce qui concerne cette occurrence, $\mathrm{X}$ est l'égal des possibles, il permet la validation de la relation prédicative. Cela n'en fait cependant pas un élément égal aux autres. La mise à niveau entre l'effectif et les possibles ne fonctionne que pour l'occurrence considérée, c'est-à-dire de façon ponctuelle.

Il s'ensuit que le co-énonciateur peut déduire de l'utilisation de even que l'élément $\mathrm{X}$ ne devrait pas, selon S, valider la RP. Donc even marque l'inclusion ponctuelle dans les éléments qui valident la relation prédicative d'un élément a priori 
situé à l'extérieur. L'énonciateur affirme l'inclusion de l'occurrence à l'intérieur du domaine notionnel de la RP, après avoir pris en compte son exclusion théorique. L'inattendu réside dans ce mouvement de balancier entre intérieur et extérieur du domaine notionnel, ou ce hiatus entre ce qui est possible et ce qui est le cas.

Cette caractéristique essentielle de even est facilement mise en relief par sa suppression dans l'exemple3: You take the test and you find that o people of color are more likely to ascribe negative attributes to darker skinned people.

Si le renvoi au domaine de référence est le même, et si la relation prédicative est la même, nous perdons tout-de-même la référence à un préalable, une préconstruction de la part de $\mathrm{S}$, de l'exclusion de people of color de la liste des $\mathrm{C}_{0}$ qui peuvent valider le prédicat ascribe negative attributes to darker skinned people. Nous perdons la référence implicite à une liste des possibles et la comparaison à cette liste que la validation impose. À ceci près que, dans l'énoncé 3 manipulé, people of color implique la prise en compte de white people, mais dans ce cas il s'agit juste de faire une comparaison entre deux groupes grâce à more likely. De ce fait, nous perdons également le hiatus dont even est la trace : hiatus entre un préalable, ce qui est possible, et un effectif, ce qui est le cas. Ce phénomène apparait de façon encore plus évidente avec la manipulation de l'exemple 2: Nancy's sister Marilyn says o her closest family members had no idea what Shenkman was doing to her. He kept her isolated.

Lorsqu'en 2, closest family members est précédé de even, le co-énonciateur comprend bien qu'il y a une mise à niveau entre les $C_{0}$ envisagés au préalable tels que friends, relatives, colleagues, etc., et celui qui est instancié par l'énonciateur, instanciation qui ne fait pas normalement partie, selon $\mathrm{S}$, des $\mathrm{C}_{0}$ qui valident cette RP. Or, si l'on supprime even, l'énoncé se transforme en une construction hors toute référence à des possibles, des $\mathrm{C}_{0}$ théoriques. Il s'agit juste de référer à ce qui est le cas, et la notion de hiatus entre le possible et l'effectif n'existe pas. Il n'y a pas de passage de la validation par E selon $\mathrm{S}$, pour réaffirmer I à l'instant $\mathrm{T}$.

\subsection{Even adverbe devant un groupe verbal}

Exemple 4: Barack Obama knows four facts about Rick Santorum that you don't. Santorum pushed for billions in wasteful pork, voting for the Bridge to Nowhere, a teapot museum, even an indoor rain forest. Santorum voted to raise the debt limit five times, increasing spending and debt by $\$ 3$ trillion. And he even voted to let convicted felons vote. (COCA)

[Barack Obama sait quatre choses sur Rick Santorum que vous ne connaissez pas. Santorum a favorisé l'investissement à perte de milliards dans le porc, fait voter pour un pont qui ne va nulle-part, pour un musée de la théière, et même pour une forêt tropicale d'intérieur. Santorum est favorable à l'augmentation par 5 du plafond de la dette, ce qui accroitrait les dépenses et la dette de trois milliards de dollars. Il a même voté pour le vote des condamnés. (Notre traduction)] 
Agnès Leroux

Exemple 5: And I feel very, very comfortable that as people continue to focus in and examine the candidates, who's the best person to win - you know, that campaign ad, first off, the felon part - Mitt Romney even admitted that that was wrong. (COCA)

[Et cela me conforte encore plus que, alors que les gens continuent à observer et à se concentrer sur les candidats (qui sera le gagnant, vous savez en fonction de la campagne d'affichage, en fonction du premier qui tire ou de celui qui a le rôle de l'escroc), Mitt Romney ait même admis que ce n'était pas correct. (Notre traduction)]

Exemple 6: Drew is an extreme altruist who donates thousands of dollars to charity and even donated one of his kidneys to a child. (COCA)

[Drew est un altruiste extreme qui donne des milliers de dollars aux œuvres de charité et qui a meme donné un rein à un enfant. (Notre traduction)]

Dans les énoncés 4 à 6 , even porte sur la relation sujet-prédicat et plus précisément sur la validation de cette relation, qui vient contrarier un pré-construit de l'énonciateur en fonction de sa connaissance du $\mathrm{C}_{0}$. C'est le prédicat qui est l'élément inattendu.

Exemple 6': Drew is an extreme altruist who donates thousands of dollars to charity and $\varnothing$ donated one of his kidneys to a child.

Cette manipulation met en évidence le fait que even permet une mise à niveau entre les validations possibles pour un $\mathrm{C}_{0}$ altruiste, selon $\mathrm{S}$, et la validation effective ponctuelle de $<$ altruist - donate one kidney $>$ pour le $\mathrm{C}_{0}$ altruist/Drew. D'où le caractère exceptionnel de cette occurrence.

Il semble d'ailleurs que lorsque even est placé en tête de prédicat et précédé de and nous ayons en plus une notion de degré, que nous pourrions formuler ainsi : un altruiste fait des dons d'argent, donner un rein est au-delà de tous les prédicats envisagés pour un altruiste, la validation de donate a kidney permet de situer l'occurrence dans le haut degré de la notion altruiste. le $\mathrm{C}_{0}$.

En 4 et 5 , le prédicat est à la limite de ce que $\mathrm{S}$ envisage comme validable pour

Ce prédicat est souvent placé, comme en 4 et en 6 , à la suite d'une liste d'éléments et il en constitue le dernier élément, nouvelle limite après la limite préenvisagée par $\mathrm{S}$.

Par conséquent, le passage par E lorsque even est adverbe devant un prédicat peut permettre de situer l'occurrence dans le haut degré du domaine notionnel.

\subsection{Even adverbe devant un adjectif à la forme comparative}

Lorsque even précède un adjectif exprimant la comparaison, il est la trace de l'exclusion du degré de qualification tel qu'exprimé dans l'énoncé, du domaine du validable construit par S. L'occurrence impose la prise en compte d'un degré a priori placé au-delà de ce que l'énonciateur envisageait comme possible. 
Exemple 7: By the time De Vries had opened the chest and Clark had been hooked up to the heart-lung machine, Clark's heart was useless. It stopped on the operating table, giving Olson and De Vries a warning that Clark was even sicker than they thought. (COCA)

[Le temps que De Vries ouvre la cage thoracique et que Clark soit branché au cœur artificiel, son propre cœur était hors service. Il a cessé de battre sur la table d'opération, avertissant ainsi que Clark était encore plus malade que ce que Olson et De Vries avaient pensé. (Notre traduction)]

Exemple 8: The show is only one of several Pacific Standard Time shows delving into the history of Chicano art in the 1960s and' 70 s, whose attitude and look seeped into mainstream art in ways only now being recognized. But the story of Asco lies even deeper, one of a subculture within a subculture, a group of artists fueled not just by their marginal existence within their city and country but by their alienation from the Chicano art movement as well. (COCA)

[Le spectacle n'est qu'un spectacle parmi tant d'autres typiques du Pacific Standard Time sur l'histoire de l'art Chicano dans les années 60 et 70, dont on reconnaît seulement maintenant qu'il imprègne l'art conventionnel. Mais l'histoire d'Asco va encore plus loin, c'est la sous-culture dans la sous-culture, un groupe d'artistes construit nonseulement autour de leur marginalité au sein de leur ville et de leur pays, mais aussi autour de leur aliénation au mouvement artistique Chicano. (Notre traduction)]

En 7, even est la trace d'un repérage à partir d'une première occurrence de $<$ Clark - be sick > d'une deuxième occurrence de cette même relation prédicative, dont le degré de qualification ne pouvait être envisagé par $\mathrm{S}$ au moment de la validation de la première occurrence.

En 8, il est prédiqué un premier niveau déjà profond, à partir duquel il est repéré un deuxième niveau pour dire qu'il relève d'une profondeur qui n'avait pas été envisagée a priori par S. Cette profondeur est difficilement concevable, mais elle est le cas.

Dans chacun de ces deux énoncés, il est prédiqué une première qualification proche de ou identique à l'adjectif déterminé par even - en 7 Clark's heart was useless, en 8 delved et seeped, évocateurs de profondeur - puis une occurrence d'un niveau au-delà de ce premier niveau préalablement envisagé comme déjà haut. Even suivi de l'adjectif à la forme comparative renvoie à la fois à la première et à la deuxième occurrence, marquant ainsi un repérage sous forme de hiatus entre les degrés de la deuxième et de la première occurrence. Le degré de la deuxième occurrence étant a priori situé par $\mathrm{S}$ à l'extérieur du domaine notionnel.

Il apparaît donc que even permet de référer au caractère inattendu d'une occurrence de la façon suivante : il permet de mettre à niveau, et donc d'induire une absence d'égalité originelle entre l'effectif et l'envisageable et ce, à l'occasion de la validation d'une seule occurrence. Le co-énonciateur peut en déduire que selon 
Agnès Leroux

l'énonciateur l'occurrence relève de l'impossible ou du non-envisagé. Lorsque even précède un verbe ou un adjectif à la forme comparative, s'ajoutent la prise en compte d'un degré, et l'expression d'une valeur extrême au-delà de laquelle nulle validation n'est envisageable selon S. Nous obtenons alors une occurrence a priori proche de la frontière extérieure du domaine notionnel de la RP, et finalement située à l'intérieur dans le haut degré.

L'espace-temps de la validation d'une seule occurrence, even marque une mise à niveau de l'intérieur du domaine notionnel et de l'extérieur. L'énonciateur construit un extérieur dont il vise a priori la validation mais cette visée est ineffective puisque l'occurrence se situe dans ce qui constitue l'intérieur du domaine notionnel.

L'inattendu n'est cependant qu'occasionnel et sa validation ne vaut que pour l'occurrence concernée.

\section{Les traductions en français : différentes façons d'envisager la référence à l'inattendu.}

Quelle est la reconstruction du sens en français? Il n'existe pas un seul, mais plusieurs marqueurs susceptibles de correspondre à la traduction de even lorsqu'il est adverbe. La différence entre ces traductions repose, comme nous allons le montrer, sur la mise en relief d'un paramètre de construction de la référence à l'inattendu au détriment des autres.

Il peut également arriver que la sélection d'un seul paramètre fasse que la référence à l'inattendu soit perdue et que la traduction pose problème.

\subsection{Recentrage sur l'intérieur du domaine notionnel}

2.1.1. Encore suivi de plus : intensité, haut degré et intégration en I d'un degré a priori placé en $E$

Encore suivi de plus marque la comparaison de deux niveaux effectifs en fonction d'un degré a priori proche de ou sur la frontière. Soit l'énoncé suivant, relevé dans le roman de John Le Carré The Constant Gardener et sa traduction par Isabelle et Mimi Perrin :

Exemple 9: He must have been clearing it, for there was not a paper in sight. Donohue looked even sicker than usual. Woodrow's wife Gloria insisted he was dying. (John Le Carré, The Constant Gardener)

[Lui que Gloria, 1'épouse de Woodrow, persistait à croire mourant avait l'air encore plus malade que d'habitude. (Isabelle et Mimi Perrin, La Constance du Jardinier)]

Dans l'énoncé 9, even suggère la possibilité du franchissement de la frontière de malade à mort, frontière évoquée par be dying. Cette frontière est en quelque sorte repoussée par even qui annonce la prise en compte en T d'un stade non envisagé de be sick lors de la validation de la première occurrence de $<$ Donobue - look sick $>$ et qui, 
selon S, pourrait être à l'intérieur de être mourant. Elle pourrait également se situer à l'intersection des domaines de be sick et be dying.

Cette insertion de deux occurrences de degrés différents mais de qualité semblable dans le domaine notionnel de be sicke, est marquée par l'opérateur encore en français. Encore permet de viser, à partir d'une occurrence, la validation de la même relation prédicative, ou d'une occurrence comparable en qualité, à un autre instant; plus signale que cette visée est effective mais à un degré supérieur à celui de la première occurrence.

Exemple 10: By some bitter inner discipline she keeps her lips tightly closed while she waits for him to make an even bigger fool of himself. Which he duly does. Royally. (John Le Carré, The Constant Gardener)

[Mue par une rigoureuse discipline intérieure, elle ne desserre pas les lèvres en attendant qu'il se rende encore plus ridicule. Ce qu'il ne manque pas de faire. Et dans les grandes largeurs. (Isabelle et Mimi Perrin, La Constance du Jardinier)]

En 10, la validation de la RP est visée, et repérée par rapport à une première occurrence, considérée comme déjà située à un haut degré. C'est encore qui est la trace de la prise en compte d'un stade antérieur à celui considéré, et à partir duquel est attribué un niveau à la deuxième occurrence. Encore laisse présager d'une occurrence identique à une occurrence préalable, mais plus montre que si la qualité est égale, le degré les rend différentes.

La combinaison encore plus marque la différence, non-envisagée a priori, de degré d'occurrences de qualité semblable, rapprochant ainsi la deuxième de la frontière du domaine notionnel. L'inenvisageable selon $S$, correspond au degré supérieur effectivement validé, à ce degré plus important que la première occurrence de la RP et qui est le cas en T. Ce haut degré n'étant pas a priori envisagé, il était placé en E, audelà de la frontière du domaine notionnel de la RP.

\subsubsection{Voire : centrage sur valeur exacte selon $S$}

La traduction par voire n'est pas très fréquente, mais elle marque selon nous un phénomène important : le passage de la ponctualité de la mise à niveau entre I et $\mathrm{E}$, à la possibilité de la généricité.

Exemple 11: There had been a plague of 'Nairobi eye' a few months back, flies that when squidged and rubbed accidentally on the skin could give you boils and blisters, and even send you blind. (John Le Carré, The Constant Gardener)

[ll y avait eu quelques mois auparavant une invasion d'onchocerques, une mouche qui, si on a le malheur de l'écraser sur la peau en frottant, peut provoquer cloques ou ampoules, voire rendre aveugle. (Isabelle et Mimi Perrin, La Constance du Jardinier)]

Avec and even send you blind, nous avons une montée dans le degré des prédicats validés par la mouche Nairobi eye dont le degré le plus important est annoncé par and: dans le domaine notionnel relatif aux effets d'un insecte, give you boils and blisters fait partie des possibles facilement envisageables mais send you blind laisse imaginer un 
Agnès Leroux

degré non envisagé ou non souhaité par l'énonciateur. Contrairement à ce préconstruit de S, send you blind est inclus dans le domaine notionnel.

$V$ oire vient du latin verus, vrai : il marque un centrage sur l'intérieur du domaine notionnel des effets de l'insecte; le centrage progressif sur rendre avengle permet de prédiquer la vraie nature de la mouche en question, en opposition à un préalable de l'énonciateur pour une mouche: not send you blind. Nous conservons une opposition entre l'envisageable selon l'énonciateur, S, et ce qui est le cas, le validé en T.

Cependant l'utilisation de could dans l'énoncé anglais pourrait laisser penser que la traduction de even par voire correspond à la réduction d'un hiatus entre l'envisageable/envisagé et une classe d'occurrences. Il ne s'agit pas de la prise en compte d'une seule occurrence qui viendrait contredire une valeur pré-construite, mais des capacités avérées du référent d'un $\mathrm{C}_{0}$ particulier. La traduction par voire permet de placer dans les occurrences possibles pour un élément des occurrences qui ne le seraient pas pour d'autres éléments de sa classe mais qui le sont de façon permanente pour l'élément considéré.

Les énoncés suivants vont permettre d'affiner l'opération marquée par voire:

Exemple 12: He understood how constrained and meagre lives could be, generation after generation. In the grand view of things, the peaceful, prosperous times England was experiencing now were rare, and within them his and Florence's joy was exceptional, even unique. (Ian McEwan, On Chesil Beach.)

[ll avait compris combien la vie pouvait être ingrate, étriquée, génération après génération. À l'échelle de l'Histoire, les temps paisibles et prospères que connaissait alors l'Angleterre constituaient une rareté, et le bonheur que Florence et lui partageaient dans ce contexte était exceptionnel, voire unique. (France Camus-Pichon, Sur la Plage de Chesil)]

Exemple 13: Edward had never cared for classical music, but now he was learning its sprightly argot - legato, pizzicato, con brio. Slowly, through brute repetition, he was coming to recognise and even like certain pieces. (Ian McEwan, On Chesil Beach)

[Edward, qui n'avait jamais beaucoup aimé la musique classique, se familiarisait à présent avec son jargon pétillant : legato, pizzicato, con brio... Lentement, à force de les entendre, il finissait par reconnaître, voire apprécier certains morceaux. (France Camus-Pichon, Sur la Plage de Chesil)]

En 12 even permet de tracer un chemin de exceptional à unique: départ du domaine notionnel lié au caractère commun : generation after generation, pour centrer sur la valeur qui convient le mieux à cette occurrence : l'unicité.

En 13 l'utilisation de and even rend la structure de l'énoncé comparable à celle de 11 : S part de l'extérieur d'un domaine notionnel donné, ici celui de like; il prédique une caractéristique, pour le référent du $\mathrm{C}_{0}$, située dans le domaine notionnel complémentaire de like - he had never cared for classical music (équivalent de not likee). A partir de ce domaine complémentaire, il s'approche progressivement de la valeur qu'il 
estime juste en passant par différents domaines notionnels qui s'éloignent de not like pour se rapprocher de like : learn, recognize, appreciate. Learn et recognize représentent des degrés sur la frontière de not like à like et appreciate le centre de la notion like.

Voire marque un centrage sur la valeur adéquate selon $\mathrm{S}$, chemin progressif de l'extérieur complet, nonP, vers l'intérieur de $\mathrm{P}$ en passant par des valeurs situées sur la frontière.

L'inattendu réside dans la construction d'un chemin entre deux complémentaires, dont l'un constitue soit le haut degré (send you blind) soit le centre, la valeur par excellence, et l'autre sa valeur inverse.

\subsection{Problématique de la frontière}

\subsubsection{Presque : station sur la frontière}

Le rapport à la frontière fait partie intégrante de la problématique de even lorsqu'il est associé à un autre opérateur, comme par exemple perbaps. Cela peut générer une traduction par presque:

Exemple 14: And there are those who go quietly. Who expect, perhaps even wait for, the police. For them, the arrival of the jeep with the flashing red light is actually a relief. (Vikas Swarup, Slumdog Millionnaire)

[Et il y en a qui suivent calmement. Qui attendent, qui guettent presque l'arrivée de la police. Ceux-là sont soulagés de voir apparaitre la jeep au gyrophare rouge. (Roxane Azimi, Les Fabuleuses Aventures d'un Indien malchanceux qui devint milliardaire)]

Who expect, perbaps even wait for the police: prise en compte de expect puis de wait qui, lui, selon S ne devrait pas valider la RP. Cependant, expect et wait ont des propriétés communes, ce qui permet d'envisager une intersection des domaines notionnels ou au moins une frontière commune.

Cette construction est comparable à celles qui ont été traduites par voire, comme le montre la possibilité de manipuler avec and:

- Who expect, and even wait for the police: nous avons alors un recentrage progressif sur la valeur exacte, de expect à wait.

En utilisant presque, pour traduire perbaps even, l'énonciateur choisit d'envisager le passage de attendre à guetter, tout en restant sur la frontière qui sépare attendre de guetter sans jamais la franchir. Perbaps marque le doute de $\mathrm{S}$ sur even : il prédique donc le doute sur la mise à niveau entre l'envisageable/le pré-construit et l'effectif. L'hypothèse marquée par perbaps porte sur le franchissement effectif de la frontière alors que presque, qui indique une prise en compte de la frontière pour ne finalement pas la franchir, représente une traduction compacte de even et perbaps. Il semblerait donc que presque marque l'opération exactement inverse de even: on envisage l'intérieur pour rester à l'extérieur. Il n'y a pas suffisamment de propriétés communes entre l'occurrence et le domaine notionnel envisagé, pour la situer à l'intérieur. L'altérité est trop importante, même si l'intérieur a été envisagé. 
Agnès Leroux

\subsubsection{Même : altérité intégrée, intersection de domaines notionnels}

Exemple 15: Almost nothing remains of District 12. A month ago, the Capitol's firebombs obliterated the poor coal miners' houses in the Seam, the shops in the town, even the Justice Building. The only area that escaped incineration was the Victor's Village. (Suzanne Collins, Hunger Games) Ill ne reste quasiment rien du district Douze. Un mois plus tôt, les bombes incendiaires du Capitole ont réduit en cendres les masures des mineurs de la Veine, les boutiques de la ville et même l'hôtel de justice. (Guillaume Fournier, Hunger Games)]

Justice building est normalement, selon $\mathrm{S}$, situé à l'extérieur de la liste des éléments qui peuvent instancier le $\mathrm{C}_{1}$ de la relation prédicative <firebomb - obliterate building $>$. Mais l'occurrence concernée permet de l'inclure dans cette liste, même si ça n'est que pour cette occurrence. Il semblerait que même fonctionne ici de façon identique. Il marque le caractère inattendu selon $\mathrm{S}$ d'une occurrence, une discordance, un hiatus entre l'attendu et l'effectif. L'effectif est alors inattendu. Donc dans ce cas, comme even, même est la trace d'un passage par E pour revenir en I.

Exemple 16: 'Mustafa would have no idea what to select,' she heard Justin reply, as firmly as before. 'And a list would be no good to him at all. Even shopping lists defeat him. I owe it to her, Sandy. It's a debt of honour and I must discharge it. Whether or not you come along.' (John Le Carré, The Constant Gardener)

[ Mustafa ne saura pas quoi prendre, entendit-elle Justin répliquer avec la même fermeté. Et ça ne lui servira à rien qu'on l'écrive, même la liste des courses le dépasse. Sandy, je dois vraiment ça à Tessa. C'est une dette d'honneur dont je dois m'acquitter, que vous m'accompagniez ou non ». (Isabelle et Mimi Perrin, La Constance $d u$ Jardinier)]

En 16, la liste des courses ne devrait pas, selon $\mathrm{S}$, instancier le $\mathrm{C}_{0}$ de la $\mathrm{RP}<\mathrm{X}-$ defeat Mustapha >, or c'est ce qui est le cas.

A. Culioli (2002) dit à propos de même que son invariant réside dans le détour. En d'autres termes, même marque la prise en compte de l'extérieur du domaine notionnel à partir de ce qui en constitue l'intérieur, pour y revenir et réaffirmer l'appartenance de l'occurrence à l'intérieur du domaine notionnel, posant ainsi la question de ses frontières. En effet, si un élément que $\mathrm{S}$ estime ne pas pouvoir appartenir à un domaine notionnel en fait finalement partie, ne serait-ce que pour une seule occurrence, cela suggère que les frontières de ce domaine sont souples et qu'elles peuvent être repoussées. Peut-on dire que le passage par E permet de ré-envisager la composition de I ?

Quel est le rapport entre E et I ?

Exemple 17: In the kitchen doorway, back arched, ears flattened, stands the ugliest tomcat in the world. "Buttercup," I say. Thousands of people are dead, but he has survived and even looks well fed. On what? $\mathrm{He}$ can get in and out of the house through a window we always left ajar 
in the pantry. (Suzanne Collins, The Hunger Games)

[Sur le seuil de la cuisine, le poil hérissé, les oreilles couchées en arrière, se dresse le plus vilain matou qui soit au monde.- Buttercup, dis-je. Des milliers de gens sont morts, mais lui a survécu. On dirait même qu'il a engraissé. De quoi s'est-il nourri ? Il peut entrer et sortir de la maison à sa guise grâce à une fenêtre que nous laissons toujours entrebâillée dans l'arrière-cuisine. Il a dû chasser des mulots. (Guillaume Fournier, Hunger Games)]

Les frontières de survivre sont revues et permettent l'intersection avec le domaine notionnel de engraisser: de survivre à avoir engraissé, même si a priori les frontières de survivre devraient s'arrêter avant engraisser, ni même évoquer la notion d'engraisser. L'utilisation de even ici permet d'associer deux domaines notionnels habituellement distincts.

Malgré la présence de and et donc une structure de surface similaire à celle relevée pour les traductions pas voire, il est impossible de le substituer à même dans cet énoncé. Il s'agit en effet de l'opération inverse : départ du centre pour envisager l'extérieur comme un extérieur et finalement déplacer la frontière jusqu'à former une intersection pour réaffirmer l'appartenance de l'occurrence à l'intérieur du domaine notionnel.

Même est donc bien la trace de la prise en compte de l'extérieur du domaine pour en affirmer l'intérieur mais sous la forme ici de l'intersection de deux domaines notionnels.

Nous conservons l'expression de l'inattendu grâce au mouvement de balancier entre intérieur et extérieur, mais ce mouvement est inverse de celui de voire. Au lieu de centrer de proche en proche, il repousse les frontières pour former une intersection.

Même postposé à un nom : la boucle d'identification

Exemple 18: Or perhaps he had been squirting mosquito spray at some Kenyan insect from which even diplomats are not immune. (John Le Carré, The Constant Gardener)

[Ou peut-être aspergeait-il d'antimoustique quelque insecte kenyan contre lequel les diplomates eux-mêmes ne sont pas immuns. (Isabelle et Mimi Perrin, La Constance du Jardinier)]

En 18 le traducteur a choisi de jouer sur l'humour contenu dans la construction de la référence à l'inattendu : even marque la prise en compte de l'extérieur du domaine notionnel des êtres humains sensibles aux piqûres d'insecte, pour prédiquer l'appartenance de l'occurrence à l'intérieur du domaine notionnel. Or, cette occurrence, diplomats, est a priori déjà placée à l'intérieur du domaine notionnel des êtres humains, mais l'immunité diplomatique en fait des êtres humains à part. Le fait d'en prédiquer la situation en I, implique que l'énonciateur les place a priori en E, pour une occurrence qui ne concerne pas l'immunité diplomatique. Dans ce cas c'est le préalable qui constitue l'inattendu.

La postposition de même au nom permet de construire une boucle réflexive terme emprunté à A. Culioli (2002, p. 17). Il y a un jeu entre le préalable du 
Agnès Leroux

co-énonciateur (qui place les diplomates en I pour cette occurrence) et celui de l'énonciateur (qui les place a priori en E) pour revenir en I, où se situe l'occurrence. Finalement, le co-énonciateur accède à la sortie du domaine notionnel telle que construite par S (évocation de l'immunité diplomatique). Il ne faut cependant pas oublier que even et même permettent de sortir les diplomates des possibles pour les faire entrer dans les effectifs uniquement pour l'occurrence dont il est question. Donc pour $\mathrm{S}$, les diplomates restent à l'extérieur du domaine notionnel des êtres humains normaux à cause de l'immunité diplomatique, ils ne sont inclus que pour les piqûres de la mouche Nairobi Eye.

Il va de soi que dans tous les énoncés où even permet l'humour, il n'est pas forcément traduit par même postposé au nom. Mais c'est ce balancier entre intérieur et extérieur, lors de la construction du préalable, pour revenir à l'intérieur qui permet l'emploi de cette structure.

\section{Quand la traduction élimine l'inattendu}

\subsection{Aussi : perte du renvoi à un pré-construit, pas de préalable en $E$}

Exemple 19: 'That necklace you're wearing,' Woodrow heard himself saying in mockchallenge as they danced. 'My grandmother gave it to my mother on her wedding day, she answered. I wear it with everything, even if it's out of sight.' 'Even in bed?' 'Depends.' (John Le Carré, The Constant Gardener)

[Ce collier que vous portez, Woodrow s'entend-il la défier par jeu tandis qu'ils dansent ensemble.

- Ma grand-mère l'a donné à ma mère le jour de son mariage, répondelle. Je le porte en permanence, même si on ne le voit pas'.

- Au lit aussi ?

- Ça dépend. (Isabelle et Mimi Perrin, La Constance du Jardinier)]

Aussi pourrait ressembler à même, mais il ne pose pas comme préalable l'exclusion de l'occurrence du domaine notionnel. Il permet de marquer l'équivalence de deux occurrences, ou l'inclusion d'une occurrence dans le domaine notionnel d'une autre, sans qu'il soit envisagé que cette deuxième occurrence pourrait ne pas lui appartenir ou que l'appartenance au domaine notionnel est exceptionnelle.

La substitution de même à aussi met cette différence en relief :

Exemple 19': Ce collier que vous portez, Woodrow s'entend-il la défier par jeu tandis qu'ils dansent ensemble.

- Ma grand-mère l'a donné à ma mère le jour de son mariage, répondelle. Je le porte en permanence, même si on ne le voit pas.

- Même au lit?

- Ça dépend. 
Par la question en même, S signifie que la validation lui semble a priori nonenvisageable.

Une part de doute est cependant présente dans l'énoncé en aussi dans la mesure où c'est une question. Mais plus que poser $\mathrm{E}$ comme préalable, la question en aussi ouvre deux chemins, l'un vers E l'autre vers I, et la traduction élimine l’inattendu.

\section{Conclusion}

En tant que trace de l'inclusion de l'occurrence d'une RP à l'intérieur du domaine notionnel, en posant comme préalable son exclusion, even permet au coénonciateur d'accéder à ce qui est considéré comme une occurrence inattendue par l'énonciateur.

Selon l'environnement contextuel de l'énoncé, ce détour par E pour affirmer I prend différentes formes et ouvre l'éventail des traductions:

- centrage progressif de E vers I sur la valeur exacte : voire;

- even + comparatif, prédication d'un degré envisagé en E, validé en I : encore plus;

- perbaps + even, passage sur la frontière de E vers I : presque;

- intersection des frontières entre le complémentaire de I et I : même;

- sortie de I pour y retourner : même postposé à un nom.

Il apparaît finalement que la conservation du hiatus entre pré-construit et effectif prime sur une traduction harmonisée de even.

Avec les marqueurs français ci-dessus, le hiatus entre les possibles selon $\mathrm{S}$ et ce qui est le cas est conservé, et au-delà des différentes façons d'envisager le rapport entre I et $\mathrm{E}$ lors de la prise en compte d'une occurrence, c'est grâce à lui que se construit la référence à l'inattendu. Si ce hiatus n'est pas conservé lors de la traduction, comme nous l'avons montré avec aussi, la référence à l'inattendu est perdue. 
Agnès Leroux

\section{Bibliographie}

Close, R. A., 1975, A Reference Grammar for Students of English, Harlow, Longman.

Culioli, A., 1990, Pour une linguistique de l'enonciation, Tome 1, Opérations et représentations, Gap, Ophrys.

Culioli, A., 1995, «Even though, even if; as though as if», Cabiers de Charles V, n¹9, Paris, Ophrys.

Culioli, A., 1999, Pour une linguistique de l'énonciation, Tome 3, Domaine notionnel, Paris, Ophrys.

Culioli, A., 2002, «A propos de même», Langue Française, No 133, p. 16-27. http://www.persee.fr/ web/revues/home/prescript/article/lfr_00238368_2002_num_133_1_1043

Ducrot, O., 1984, Le dire et le dit, Paris, Editions de Minuit, p. 33-46.

Ducrot, O., 1980, Les mots du discours, Paris, Editions de Minuit.

Gauthier, A., 1997, «Even, just et le domaine notionnel », La Notion, Paris, Ophrys, p. 121128.

GrizE, J. B., 1997, Logique et langage, Paris, Ophrys.

Huddleston, G., and Pullum, G. K., 2005, A Student's Introduction to English Grammar, Cambridge, Cambridge University Press, p.122-125.

\section{Références du corpus}

Corpus électronique de littérature contemporaine créé par le groupe LIDIL/IMAGER de l'UPEC.

Corpus of Contemporary American English (COCA) : http://corpus.byu.edu/coca/ 